Didáctica Geográfica n 19, 2018,pp. 149-167

ISSN: 0210-492-X

D.L: M-3736-2014

\title{
PERCEPCIÓN DEL ALUMNADO DEL IES "EL TABLERO" (CÓRDOBA) SOBRE LA ENSEÑANZA Y EL APRENDIZAJE DE LA GEOGRAFÍA
}

José Manuel Lara Fuillerat ${ }^{1}$; José Moraga Campos²

Recibido: $12 / 04 / 2018$

Aceptado: 29/06/2018

\section{RESUMEN:}

En este artículo pretendemos analizar la percepción del alumnado del IES El Tablero (Córdoba) sobre el proceso de enseñanza y aprendizaje de la asignatura de Geografía en los diferentes niveles en los que se imparte. Para ello, hemos utilizado un cuestionario anónimo que ha sido cumplimentado por una gran parte del alumnado del centro. Los resultados y el análisis de los mismos nos servirán para afianzar, mejorar o replantear la propuesta didáctica de esta materia.

\section{Palabras Clave:}

Enseñanza, Aprendizaje, Geografía, Bachillerato, Enseñanza Secundaria.

\section{Abstract:}

In this article we intend to analyze the perception of the students of the IES El Tablero (Córdoba) about the teaching and learning process of the subject of Geography in the different levels in which it is taught. For this, we have used an anonymous questionnaire that has been completed by a large part of the students of the center. The results and the analysis of them will serve to strengthen, improve or rethink the didactic proposal of this subject.

1 IES El Tablero (Córdoba). josemanuel.lara@ieseltablero.es

${ }^{2}$ Centro del profesorado de Córdoba. jose.moraga.edu@juntadeandalucia.es 


\section{KEYWORDS:}

Teaching, Learning, Geography, Baccalaureate, Secondary Education.

\section{RÉSUMÉ:}

Avec cet article, nous avons l'intention d'analyser la perception des étudiants de l'IES El Tablero (Cordoue) sur le processus d'enseignement et d'apprentissage du sujet de géographie dans les différents niveaux dans lesquels il est enseigné. Pour cela, nous avons utilisé un questionnaire anonyme qui a été rempli par une grande partie des étudiants du centre. Les résultats et l'analyse de ceux-ci serviront à renforcer, améliorer ou repenser la proposition didactique de ce sujet.

\section{MotS-ClÉs:}

Enseignement, Apprentissage, Géographie, Baccalauréat, Enseignement Secondaire.

\section{Planteamiento INICIAL}

La finalidad esencial del este artículo es proseguir con la labor de indagación emprendida en una investigación previa sobre la actitud y la percepción que el alumnado del IES El Tablero de Córdoba mostraba sobre la enseñanza de la Historia en los diferentes niveles en que se impartía (Capdevilla Gómez, A. et alii, 2016). En esta ocasión queremos descubrir cuál es la percepción que tienen esos mismos alumnos/as sobre la asignatura de Geografía, tanto en Secundaria $\left(1^{\circ}, 2^{\circ}\right.$ y $3^{\circ}$ de ESO) como en Bachillerato (Geografía de España en $2^{\circ}$ de Bachillerato). Para ello, y con el propósito de propiciar posteriores comparaciones, usamos la base del cuestionario anterior adaptándolo a las peculiaridades de esta materia académica ${ }^{3}$.

Antes de escrutar los resultados obtenidos, hemos querido efectuar una breve prospección por algunas de las referencias científicas que han tratado este mismo objeto. La primera apreciación que hemos recibido es que existen muy pocos trabajos que vayan encaminados en esta misma dirección. La mayor parte de la investigación práctica examinada se orienta en algunas de las siguientes líneas:

$1^{\circ}$ Pruebas de nivel de conocimiento geográfico (¿qué sabes de la Geografía de...? Localiza en un mapa,...).

\footnotetext{
${ }^{3}$ Debido a limitaciones de extensión, no se ha podido incluir en este artículo ni el cuestionario ni el anexo estadístico y gráfico. No obstante, ambos se pueden descargar directamente desde el siguiente enlace: https:/www.dropbox.com/s/jyqyz53gh739kj4/Lara_Moraga_Percepcion\%20 Geografia_Cuestionario\%20y\%20anexo\%20estadistico.pdf?dl=0
} 
$2^{\circ}$ Estudios sobre las dificultades de aprendizaje de la Geografía.

$3^{\circ}$ Justificaciones desde el profesorado sobre la necesidad de enseñar Geografía.

$4^{\circ}$ Metodologías de enseñanza y aprendizaje de la Geografía.

$5^{\circ}$ Ideas previas del alumnado sobre aspectos concretos de contenido geográfico.

$6^{\circ}$ Estudios sobre el entorno del alumnado (con participación o no del alumnado en el diseño de la actividad)

Hace ya algún tiempo, el profesor X.M. Souto González (1998, p. 60-66) constató la carencia de estudios sobre la percepción que tiene el alumnado sobre la Geografía como materia de estudio. En la mayor parte de los trabajos que examinó al respecto, el alumnado manifestaba una nítida concepción descriptiva de la materia (principalmente relacionada con los accidentes geográficos), con cierta confusión con lo tratado por la Historia y, en general, un aprendizaje poco significativo. Esto le llevaba a concluir, por un lado, que existía un bajo nivel de conocimiento de esta materia y, por otro, a la necesidad de estudiar conceptos de Geografía Física en edades más tardías, ya que la complejidad de éstos los hacía inabarcables a edades tempranas. No obstante, Souto argumentaba que la percepción negativa de la Geografía no era cuestión de nivel, ni de incorporación tardía de conocimientos geográficos, sino que se debía a que "los alumnos desprecian u olvidan aquello que creen que no es significativo para su aprendizaje" (Souto González, 1998, p. 62). Concluía que era posible cambiar la sentimiento de apatía y rechazo hacia el estudio de la Geografía y, para ello, apostaba, en primer lugar, por más tiempo escolar, esto es, más dedicación a esta materia y, en segundo lugar, por mejorar la formación inicial del profesorado tanto en contenidos como en metodología. Se trataba de formular una propuesta motivadora, basada en el estudio de los problemas sociales y ambientales y en metodologías activas para que el alumnado considere provechoso el estudio de una asignatura como la Geografía. Y esto eran conclusiones y análisis que se realizaban en 1998. Veinte años después se sigue hablando de manera recurrente de este tema y, por lo que a nosotros respecta, coincidimos totalmente tanto en el análisis como en las conclusiones, lo que viene a sugerirnos la idea de que poco hemos avanzado en la actitud del alumnado hacia nuestra disciplina.

Para contextualizar nuestra aportación a esta temática, debemos apreciar la coyuntura en la que nos encontramos, un contexto de crisis y transformación del paradigma educativo. Desde principios del presente siglo, el proceso de enseñanza-aprendizaje se ha orientado, al menos en sus vertientes teórica y normativa, hacia el concepto de competencia clave. Este enfoque estima centrar el proceso educativo en el aprendizaje del alumno/a y superar la enseñanza tradicional basada en el profesorado, que tenía que 
enseñar, y en los contenidos, que se debían transmitir. Los inconvenientes derivados de este paradigma son numerosos, destacando, entre ellos, los siguientes:

- Los conocimientos asimilados son rápidamente olvidados por el alumnado y, en la mayoría de los casos, se desactualizan de manera vertiginosa.

- Existe una cierta desmotivación por parte del alumnado hacia esta metodología que se basa esencialmente en la memorización. Es el caso de la Geografía, a la que, mayoritariamente, relacionan con la memorización de accidentes geográficos, datos, conceptos descontextualizados, etc., válida para asistir a concursos televisivos, pero de poca utilidad práctica para nuestro alumnado.

- No existe una integración de los conocimientos adquiridos en las distintas materias.

- Utilización de metodologías predominantemente transmisivas en la que el alumno/a asume un rol muy pasivo.

Con el nuevo enfoque competencial se pretende inculcar en el alumnado toda una serie de habilidades, destrezas y conocimientos, esto es, competencias, que favorezcan su desenvolvimiento en un mundo dinámico, cambiante, líquido, como es el actual. El proceso de enseñanza-aprendizaje se centra en el alumnado y en el "aprender a aprender". En palabras del profesor Tourón, este enfoque "permite a los estudiantes aprender lo que tienen que aprender en el modo en el que lo aprenden mejor" (Touron, 2017). Se pretende que el alumnado se ejercite en construir, crear, comprender, debatir, interactuar y aprender. En este sentido, debemos cuestionar lo que los centros escolares imparten, en contraposición a las herramientas y maneras de actuar e instruirse de muchos de nuestros alumnos en entornos informales y no formales de aprendizaje.

Todo ello comporta un cambio sustancial: el profesorado debe reinterpretar su función dado que este planteamiento supone la pérdida de su papel preponderante, central, en aras de un mayor protagonismo del alumnado en su propio proceso de aprendizaje. El profesor/a pasa a desempeñar un rol de guía, apoyo, acompañamiento del alumno/a en tanto que éste debe asumir un mayor grado de autonomía en la organización de su aprendizaje, en su capacidad de tomar decisiones y asumir responsabilidades, en el autoevaluarse y supervisión de su aprendizaje. Sintéticamente, ésta sería la base del nuevo enfoque competencial que se pretende implantar en el sistema educativo tanto español como europeo. No podemos extendernos más en el análisis de este concepto. Para ello creemos más acertado remitirnos a la abundante literatura pedagógica existente $^{4}$. No obstante, creemos necesario inferir cómo este nuevo enfoque competencial

${ }^{4}$ Para un análisis general y básico del concepto de competencias recomendamos: Moya Otero, J.; Luengo Horcajo, F. (coord.) (2011): Teoría y práctica de las competencias básicas, Barcelona: 
puede incidir en la enseñanza y aprendizaje de la disciplina geográfica en Enseñanza Secundaria y Bachillerato y, al mismo tiempo, qué puede proporcionar esta disciplina a la nueva configuración del sistema educativo y, lo que es más trascendental, qué le puede aportar a nuestro alumnado, desde su propio punto de vista, puesto que, desde el lado del profesorado sí tenemos más o menos claro su utilidad pedagógica tal y como se ha expuesto en diversos estudios al respecto ${ }^{5}$. A manera de ejemplo, podemos referir las conclusiones a las que llegó, hace algunos años, Fernando Arroyo Illera (1994, p. 43 y ss.) quien sintetizaba en cuatro los valores que puede aportar la educación geográfica:

1. La Geografía potencia y sistematiza las aptitudes de percepción espacial, conocimiento del territorio, identidad del hombre con su medio, arraigo, etc.

2. La enseñanza de la Geografía incide en la transmisión de una herencia cultural propia.

3. La enseñanza de la Geografía pretende ser la de un determinado valor ético, que configure toda una actitud ante el entorno de la vida del hombre y ante las relaciones de ambos.

4. La Geografía debe jugar un cometido primordial en la formación de una competencia espacial que permita al grupo de ciudadanos desempeñar la función que le corresponde en el actual sistema socioeconómico.

Además, añade este autor, la Geografía proporciona (o debe proporcionar) una serie de destrezas comunes a otras disciplinas como pueden ser la capacidad de relación y observación, la facilidad de expresión y, muy significativamente, la abstracción y la reflexión teórica. Pero también aporta otras destrezas y aptitudes que le son propias o más cercanas como la graficidad, la visualidad y la capacidad de observación, la capacidad de medición y valoración de tamaños y magnitudes, tanto espaciales como sociales, la valoración del trabajo de campo, el contacto vivo y directo con los hechos y problemas geográficos y, finalmente, el manejo de tecnología audiovisual e informática (TIC y TAC). ${ }^{6}$

Graó; Escamilla, A. 2011. Las competencias en la programación de aula (vol. II): educación secundaria (12-18 años), Barcelona: Graó; Zabala Antoni, 2007. 11 ideas clave: cómo aprender y enseñar competencias. Barcelona: Graó; Gimeno Sacristán, J. Comp., 2008. Educar en competencias, ¿qué hay de nuevo? Madrid: Ediciones Morata.

5 Podemos ampliar este punto a partir del Informe sobre la situación de la Geografía en Secundaria y Bachillerato que en 2013 elaboraron para la A.G.E. Isaac Buzo y Paloma Ibarra. Igualmente, podemos examinar la reciente Declaración Internacional sobre Educación Geográfica elaborada en 2016 por la Comisión de Educación Geográfica de la Unión Geográfica Internacional (ver bibliografía).

${ }^{6}$ Una actualización de estas aportaciones de la Geografía a la formación de nuestro alumnado se puede ver en los capítulos dedicados a ésta y redactados por Xosé Manuel Souto en Prats (2011). 
Más recientemente, el profesor De Miguel (2016, p. 11-39) concibe un novedoso análisis de las aportaciones y posibilidades que proporciona la Geografía para su aprendizaje vinculándolo con conceptos pedagógicos muy actuales como los estilos de aprendizaje (aunque esta teoría sea muy cuestionable), la neuroeducación, etc.

En definitiva, podemos sugerir que la contribución de la ciencia geográfica a la formación del alumnado/a de Secundaria es muy apreciable y, siguiendo a N. J. Graves (1989, p. 20), afirmar que "Ningún habitante de esta tierra conseguirá una educación completa ni se convertirá en ciudadano autónomo y responsable mientras no haya adquirido una educación geográfica por no decir "reflejos" geográficos".

Compartimos estos planteamientos, pero creemos, igualmente, que es preciso hacer más "pedagogía" sobre las bondades de la Geografía como disciplina escolar, perseverar más en los factores diferenciales de dicha disciplina como conocimiento esencial y útil para la formación integral de nuestro alumnado e incidir en la actualización tanto de contenidos conceptuales (que pasaría por estudiar aspectos relacionados con la Geografía cultural, Geoecología, Paisaje y Geopolítica), como procedimentales (técnicas geoespaciales, graficidad, observación, geolocalización y cartografía) y metodologías activas (aprendizaje basado en proyectos o problemas, trabajo de campo, aprendizaje cooperativo, etc.). Además de todo lo anterior, tendríamos que añadir la necesidad de formar a nuestro alumnado en competencia espacial, fundamental en el mundo actual, pero apenas desarrollada en el mundo escolar.

En los últimos años percibimos una marcada preocupación, desde la didáctica de la Geografía, por conocer cómo aprenden los alumnos y alumnas, y más concretamente por evidenciar los problemas o dificultades de aprendizaje. Esa preocupación podría deberse a lo que en su día indicó Mario Carretero (1997.p. 17) al hablar de "dificultades de aprendizaje". Con este concepto se hace referencia a toda una serie de dificultades que presenta el alumno/a, que no pertenece al grupo definido como de necesidades educativas especiales, para alcanzar los objetivos y competencias establecidos en el currículum oficial, así como para asimilar los contenidos establecidos para cada nivel educativo y materia en Secundaria. Según esta definición, podemos establecer dos tipos de problemas en el aprendizaje de la Geografía:

1. Problemas comunes a otras disciplinas sociales (extensibles a otras disciplinas que no pertenecen a este ámbito).

2. Problemas derivados de la propia disciplina geográfica.

Respecto a los primeros Ángel Liceras (2000) señalaba los siguientes:

a) Factores de índole personal. Incumben al propio alumno/a y a su manera de afrontar el estudio. Dentro de ellos, se pueden distinguir varios subtipos: 
i) Factores cognitivos, se vinculan con la capacidad de aprender del alumno/a, su atención, su madurez cognitiva, su capacidad de memorización y de comprensión de conceptos abstractos.

ii) Factores motivacionales, esto es, la motivación del alumno/a hacia el aprendizaje.

iii) Factores pedagógicos como los métodos de enseñanza, el currículum, los recursos materiales disponibles, la adecuación de espacios y tiempos, dificultades de comprensión, vocabulario, interacción con otros alumnos/as, el profesorado, etc.

b) Factores de índole socioeconómica, se refieren al entorno familiar, a las condiciones socioculturales y económicas y a las escolares.

c) Factores específicos de las ciencias sociales, como, por ejemplo:

i) Uso abusivo de la memorización no comprensiva.

ii) Cambio de un modelo de enseñanza descriptiva a otro explicativa.

iii) Dificultad para realizar un currículum interdisciplinar que sirva para proporcionar una visión de la realidad social más amplia e inclusiva.

iv) Falta de interés del alumnado por cuestiones sociales.

v) Las dificultades derivadas de la propia naturaleza de los hechos sociales.

vi) Las ideas previas que tiene los alumnos/as de los hechos sociales.

En cuanto a los problemas derivados de la propia enseñanza de la Geografía cabe enfatizar los siguientes:

1. Predominio de una visión académica de la materia.

2. Dificultad de comprensión del concepto de espacio geográfico.

3. Dificultades para la comprensión e interpretación de mapas.

4. Problemas de comprensión de los principales conceptos geográficos.

5. Ideas previas del alumnado sobre la enseñanza de la Geografía.

6. Dificultad para relacionar hechos, causas y fenómenos de índole espacial.

Algunos de estas dificultades las vamos a ver reflejadas en los resultados del cuestionario que hemos utilizado con nuestro alumnado.

Para finalizar esta introducción y antes de pasar a mostrar los resultados de dicho trabajo, queremos especificar que uno de los aspectos cruciales para nosotros 
en relación con este tipo de estudios es el de su utilidad real, práctica, es decir, a parte de su posible interés académico, su realización debe provocar en el profesorado un proceso de reflexión sobre su propia práctica docente y, por extensión, sobre la mejora del proceso de enseñanza y aprendizaje de nuestra disciplina. Sin duda, la autoevaluación es necesaria de cara a la mejora de nuestra praxis pero no podemos obviar la heteroevaluación inversa, esto es, la que realiza el alumno/a sobre el profesor/a. Estamos totalmente convencidos que, bien planteada y preparada, la evaluación (y no sólo la toma de opinión o la realización de encuestas de satisfacción) del alumnado nos puede aportar una interesante retroalimentación. Es más, juzgamos que debería formar parte del diseño de situaciones de aprendizaje habituales. El alumno/a cobra más protagonismo en su proceso de aprendizaje y, como parte del mismo, está el evaluar cómo se ha desarrollado dicho proceso diseñado esencialmente por el profesor/a.

Sostenemos que debemos partir siempre del resultado de las evaluaciones realizadas, de las percepciones propias o ajenas sobre cómo aprenden nuestros alumnos y alumnas o sobre la eficacia de las metodologías puestas en práctica durante el proceso. Sin embargo, en la realidad, rara vez se tiene en cuenta la opinión del alumnado. Esto supone, sin duda alguna, una cierta pérdida de capacidad de mejora, puesto que no consideramos todas las variables posibles que afectan a dicho proceso y, además, perdemos el punto de vista del protagonista esencial de nuestro trabajo. Se trata, por ende, de considerar como una variable más a tener en cuenta en los procesos de evaluación la valoración que realizan nuestros alumnos y alumnas. El análisis de las opiniones de los alumnos/as, lo que creen o sienten, lo que realmente consideran importante, relevante o útil, puede servirnos para enriquecer nuestra labor docente, si bien debemos considerar, igualmente, los límites que esta práctica puede significarnos.

En definitiva, afrontamos este trabajo como una experiencia de indagación y mejora de nuestra práctica docente y del proceso de enseñanza y aprendizaje de la Geografía, una materia que consideramos imprescindible pero cuya enseñanza es manifiestamente mejorable.

\section{CONTEXTO}

El cuestionario que hemos manejado para este trabajo ha sido cumplimentado por los alumnos/as del I.E.S. "El Tablero" de Córdoba. Se trata de un centro de tipo medio, cuyo alumnado procede de siete zonas urbanas del Norte y Noroeste de Córdoba. Es un alumnado social y económicamente heterogéneo, mayoritariamente de clase media y media-baja, con importante presencia de profesiones liberales -es significativo el número de docentes-, con un nivel cultural en el que resaltan los estudios secundarios o universitarios. Hay que manifestar que, en general, las familias están muy implicadas 
en el proceso formativo de sus hijos e hijas. No obstante, en el primer ciclo de secundaria se detecta un cierto problema de alumnado absentista o de asistencia irregular.

Respecto a la composición de los grupos, en el Primer Ciclo de Secundaria $\left(1^{\circ}\right.$ y $2^{\circ}$ de ESO) existen 4 grupos por nivel, con una ratio en torno a los 25-30 alumnos/as, con un Programa de Bilingüismo. Son los cursos con mayores problemas de convivencia y máxima heterogeneidad socioeconómica y cultural, con algunos estudiantes que pertenecen a familias al límite de la exclusión y algunos procedentes de centros de menores. Para atender a este alumnado se cuenta con aulas de apoyo inclusivo y profesorado especialista en educación compensatoria.

El Segundo Ciclo de Secundaria lo componen 6 grupos, 3 por nivel, con una ratio sobre los 25 alumnos/as. En este ciclo los problemas de disrupción son sensiblemente menores respecto al ciclo anterior.

En cuanto al Bachillerato, actualmente contamos con tres grupos de $1^{\circ}$ y tres de $2^{\circ}$, la ratio de alumno/aula se incrementa sensiblemente, acercándose a los 30-35 matriculados por aula. En esta etapa, al alumnado procedente de la Secundaria del propio centro se le suma un importante número de alumnos/as procedentes de centros privados con diferentes niveles formativos.

En cuanto al contexto curricular en el que nos movemos, de manera sintética, podemos recordar que, por la nueva ley educativa LOMCE, los alumnos/as van a estudiar contenidos geográficos en los tres primeros cursos de la ESO y en $2^{\circ}$ de Bachillerato.

En $1^{\circ}$ de ESO se estudian aspectos relacionados con la Geografía Física como son el Planeta Tierra, sus elementos constitutivos (hidrosfera, geosfera y atmósfera), nociones elementales de Geomorfología y Climatología, los paisajes del mundo y los problemas medioambientales. En $2^{\circ}$ de ESO se desarrollan contenidos de Geografía Humana, Geografía económica, urbana y de la población. Tanto en $1^{\circ}$ como en $2^{\circ}$ la Geografía comparte tiempo con la Historia y, en la mayor parte de los casos se suele priorizar el temario de contenido histórico sobre el geográfico.

En $3^{\circ}$ de ESO el currículo se centra exclusivamente en Geografía, especialmente, Geografía Económica (aspectos relacionados con las actividades económicas) así como medio ambiente, desarrollo sostenible y contenidos relativos a la Geografía Política (globalización, sistemas políticos, la U.E., etc.)

En $2^{\circ}$ de Bachillerato, la Geografía se centra en España, tanto física como humana. Se trata de una optativa dentro del itinerario de Humanidades y Ciencias Sociales. El rasgo más destacable de esta asignatura es su excesiva extensión y complejidad lo que la hace poco atractiva para el alumnado. En los últimos años, el número de alumnos que cursan Geografía de $2^{\circ}$ Bachillerato de Humanidades y Ciencias Sociales en nuestro centro 
ha disminuido a causa de las modificaciones legislativas, el descenso del número de unidades que hemos experimentado en Primero y, sobre todo, las dificultades inherentes a esta disciplina.

\section{METODOLOGÍA DESARROLLADA}

La base metodológica de este trabajo ha sido una encuesta similar a la utilizada en la investigación precedente sobre la actitud y percepción de la Historia en la E.S.O. y Bachillerato. Se ha desarrollado durante los meses de enero y febrero de 2017 en los niveles de $1^{\circ}, 2^{\circ}, 3^{\circ}$ de ESO y en la materia optativa de Geografía de España en $2^{\circ}$ de Humanidades y Ciencias Sociales. Ha sido completada por un total de 247 alumnos/as. A tal fin se ha utilizado la herramienta Formularios que se incluye en la aplicación de Google Drive. La encuesta estaba estructurada en tres secciones:

$1^{\circ}$ Cuestiones generales sobre hábitos de estudio;

$2^{\circ}$ Métodos y técnicas de estudio en Geografía;

$3^{\circ}$ Actitud frente al estudio de la Geografía.

Si bien los respuestas obtenidas en el primer apartado son de gran interés para nosotros, dado que nos proporcionan ideas genéricas sobre la manera de afrontar el proceso de aprendizaje por parte del alumnado, hemos creído oportuno obviar su análisis en este artículo por motivos de extensión y centrarnos en las otras dos secciones cuyo análisis nos proporcionará una visión genérica de la actitud y la percepción que manifiesta este alumnado sobre la Geografía. Indudablemente, se trata de un estudio de un caso concreto, particular, de ahí que las conclusiones que se extraigan no pueden ser generalizables, ni mucho menos. Sería preciso extender este estudio a otros centros y realizar un análisis mucho más preciso para detectar factores y elementos que pueden incidir en la percepción del alumnado respecto a nuestra disciplina. Juzgamos igualmente que hubiera sido interesante confrontar estos mismos resultados con los obtenidos al preguntar sobre la Historia. Aunque estamos trabajando en ello, aún no podemos exponer las conclusiones que serán objeto de otro artículo.

\section{RESULTADOS}

Iniciamos este análisis de resultados por la sección segunda del cuestionario preguntando sobre las técnicas de estudio preferidas a la hora de afrontar el estudio de la materia de Geografía. En general, se observa una preferencia por el uso del libro de texto (33'06\% del total de encuestados), seguido por los apuntes (31'42\%) y los resúmenes $(23 \%)$, mientras que Internet y las lecturas complementarias tienen un menor 
uso $^{7}$. En la ESO observamos un uso primordial del libro de texto, incluido dentro del Programa de Gratuidad de la Junta de Andalucía. Por niveles, en $1^{\circ}$ de ESO se combinan el libro de texto con los resúmenes y los apuntes de clase; en $2^{\circ}$ y $3^{\circ}$ de ESO, se señalan, primero los apuntes y después el libro de texto y a cierta distancia los resúmenes. Esta distribución de técnicas y métodos de estudio está marcada, fundamentalmente, por la praxis docente del profesorado. En $2^{\circ}$ de Bachillerato, destacan los apuntes y los resúmenes, conjuntamente con informaciones obtenidas en Internet. Esto se debe a que en nuestro Centro no tenemos establecido ningún libro de texto oficial como referente, siendo el profesorado el que elabora los materiales de clase que el alumno/a trabaja para la adquisición de sus aprendizajes. Estos materiales se adaptan a los requerimientos de las Pruebas de Evaluación del Bachillerato para el Acceso a la Universidad (PEBAU) y se procura actualizar tanto los contenidos como las prácticas con los datos más recientes, siendo de libre acceso para el alumnado, y publicado en el apartado materiales de la web del Departamento de Geografía e Historia.

En el siguiente cuadro aparece recogido el uso que se ha manifestado de las diferentes técnicas y métodos de estudio considerados.

TABLA N $^{\mathrm{o}} 1$. Técnicas de estudio utilizadas por el alumnado de Geografía.

\begin{tabular}{|l|c|c|c|c|c|c|}
\hline & \multicolumn{7}{|c|}{ APARTADO DE LA ENCUESTA EN \% (1) } \\
\cline { 2 - 7 } & $\mathbf{2 . 2}$ & $\mathbf{2 . 3}$ & $\mathbf{2 . 4}$ & $\mathbf{2 . 5}$ & $\mathbf{2 . 6}$ & $\mathbf{2 . 7}$ \\
\hline Siempre & 48,98 & 20,41 & 34,84 & 37,70 & 7,79 & 28,93 \\
\hline A menudo & 19,18 & 24,49 & 23,36 & 26,23 & 24,59 & 42,15 \\
\hline A veces & 22,45 & 37,14 & 25,41 & 21,72 & 40,16 & 23,14 \\
\hline Nunca & 9,39 & 17,96 & 16,39 & 14,34 & 27,46 & 5,79 \\
\hline
\end{tabular}

Apartado 2.2: Lectura previa del tema. 2.3 Esquemas, mapas conceptuales...

2.4 Subrayado. 2.5 Resúmenes. 2.6 Otras fuentes de información. 2.7 Memorización de la información.

\footnotetext{
Sobre el uso del libro de texto en Geografía se recomienda ver los trabajos de Rivas Barrós, S. y Benso Calvo, M.C. 2002: La geografía del libro de texto en la enseñanza secundaria. Uso y producción de manuales en el bachillerato gallego decimonónico, Historia de la educación: Revista interuniversitaria, $\mathrm{n}^{\circ}$ 21, págs. 153-175, (https://goo.gl/xkvcyx); y Gouveia, E.L.-Montiel, K. y Bejas, M. 2005: Uso y abuso de los libros de texto en la enseñanza de la Geografía, Geoenseñanza. Vol.10 (2). Julio-diciembre, págs. 173-186 (https://goo.gl/gipCfU). [Consulta 6/3/2018]).
} 
En general, los alumnos/as suelen adoptar diversas técnicas de estudio al mismo tiempo; pueden leer los contenidos previamente a ser trabajados en clase (cuando lo recomienda el profesor), estrategia de aprendizaje que facilita la asimilación y comprensión de la asignatura y que nos puede brindar interesantes situaciones de aprendizaje (Nin y Leduc, 2013).

Esquemas, organigramas o mapas conceptuales no se usan de forma tan preponderante. Son técnicas explotadas más en los cursos superiores que en los iniciales y, aunque existen diferencias muy apreciables entre dichos métodos, se emplean más los esquemas que los mapas conceptuales que requieren de una práctica más intensa y un adiestramiento que, habitualmente, no suele enseñarse, ya que, en muchas ocasiones, tampoco es manejada con preferencia entre el profesorado (López Fecal, 1992, p. 31-35)

Otra de las técnicas asiduamente utilizadas en clase suele ser el subrayado de la información. Sin embargo, en esta ocasión, hemos evidenciado que no era de las más usadas, variando desde el 55,10\% de $1^{\circ}$ de ESO al $75 \%$ de $2^{\circ}$ de Bachillerato. Esta variación es consecuencia del propio programa de gratuidad de libros de texto que impide subrayar el texto para no deteriorarlo.

La realización de resúmenes en la materia de Geografía es una de las técnicas preferidas por el alumnado, especialmente en $1^{\circ}$ de ESO y $2^{\circ}$ de Bachillerato.

Sobre el manejo de fuentes complementarias de información diferentes al libro de texto o apuntes del profesorado, se ha verificado una práctica limitada y ocasional, siempre más destacada en los cursos superiores que en los iniciales, como cabía esperar al implicar mayor grado de autonomía por parte del alumnado.

En cuanto al uso de la memoria en la adquisición de los contenidos hemos observado un contundente resultado: el $71^{\prime} 08 \%$ de los encuestados ha contestado positivamente, ("Siempre” y “A menudo"), con un 23'14\% que lo hace esporádicamente y un 5'79\% que no utiliza esta técnica. Estos resultados nos sugieren que la memorización de contenidos es, como indicamos anteriormente, la técnica de estudio más utilizada, aunque es la que menos aprendizaje significativo puede generar. Debemos especificar, no obstante, que este rasgo no solo afecta a la materia de Geografía sino también a la Historia, como se verificó en el trabajo anterior y, nos atrevemos a atestiguar, que en la mayoría de las asignaturas. Como expresa J.A. Santiago Rivera "La vigencia de la memorización, la repetición y la fijación de nociones y conceptos, constituye una situación preocupante en la enseñanza de la geografía [...] porque contrasta esta forma de aprender tan tradicional con el desenvolvimiento de emergentes circunstancias de escenarios geográficos complejos e inciertos. Significa entonces que los aprendizajes escolares resultan contradictorios con la forma como se dan a conocer en los medios de comunicación social y en la discusión cotidiana, los nuevos temas geográficos. De allí la 
exigencia de una acción educativa remozada que forme a los ciudadanos como actores protagonistas críticos de los acontecimientos y no como espectadores apáticos, sumisos y alienados" (Santiago Rivera, 2004),

La cuestión 2.8 (Por lo general, ¿comprendes lo que estudias?) nos aporta una información bastante sugestiva. La dificultad de comprensión de los contenidos geográficos va incrementándose progresivamente: un $53 \%$ en el alumnado de $1^{\circ}$ de ESO comprende siempre lo que estudia en tanto que tan sólo el 18,75\% de Bachillerato lo hace. Este es un dato muy significativo, puesto que vendría a aportar una primera causa de la falta de apego hacia nuestra disciplina en el Bachillerato: a los alumnos y alumnas les cuesta asimilar en su totalidad los contenidos de Geografía. Según Vera y Cubillos (2010, p. 14) esto hecho se debería a dos factores fundamentalmente: por un lado, al grado de complejidad de estos mismos conceptos y, por lo tanto, su comprensión está sujeta a las capacidades y al desarrollo cognitivo del alumnado, y, por otro, al papel del docente como principal agente facilitador del aprendizaje del alumnado.

Posteriormente, hemos inquirido sobre el tiempo que dedican semanalmente a la materia/contenidos de Geografía. Predomina la franja de una o dos horas a la semana (41'22\%), seguida de la franja de tres a cuatro horas (31'24\%). Las respuestas varían por niveles: en $1^{\circ}$ de ESO predomina el intervalo de tres a cuatro horas mientras que en $2^{\circ}$ y $3^{\circ}$ de ESO, se señalan menos horas de estudio; en $2^{\circ}$ de Bachillerato, se incrementa levemente, posiblemente al tratarse de una materia específica y optativa con unos requerimientos curriculares de mayor complejidad. Un dato bastante negativo que hemos podido constatar es el elevado número de alumnos que en $3^{\circ}$ de ESO (15'52\%) y $2^{\circ}$ de Bachillerato $\left(12{ }^{\prime} 5 \%\right)$ han manifestado no estudiar nada esta asignatura, el doble de lo que ocurre en los grupos de $1^{\circ}$ y $2^{\circ}$ de ESO. Nos encontraríamos ante alumnos/as que no muestran ningún interés por esta materia o bien que han decidido abandonarla.

Siguiendo con esta variable temporal, hemos preguntado sobre la necesidad de dedicar más o menos tiempo que otras materias para la preparación de los contenidos geográficos. En general, se ha constatado que se requiere un mismo lapso de tiempo que otras asignaturas, salvo en Bachillerato, donde sí hay una mayoría de alumnos que contestan positivamente a esta necesidad de mayor tiempo en su preparación de la asignatura, posiblemente por la complejidad de la propia materia, como ya hemos indicado anteriormente.

El tercer apartado del cuestionario es el más interesante para nosotros al tratar de indagar la actitud del alumnado frente a la Geografía como asignatura. Un resumen de los resultados puede ser visto en la Tabla ${ }^{\circ} 2$. Comenzamos preguntando si le gustaba estudiar Geografía. Un 56'14\% de los encuestados respondía "Mucho" y "Bastante". Esta cifra es inferior a la que señalaron en la encuesta sobre Historia y, además, está irregularmente repartida entre los distintos niveles educativos: mientras que en $1^{\circ}$ y $2^{\circ}$ de ESO se halla por 
encima de esta media, en $3^{\circ}$ de ESO y $2^{\circ}$ de Bachillerato es inferior al cincuenta por ciento, precisamente en los cursos en los que la Geografía no comparte tiempos con la Historia. En estos datos, tal y como concluiremos, estimamos posible que influyan elementos de carácter personal respecto al profesorado que imparte la asignatura.

TABLA N $^{\circ}$ 2. Actitud frente al estudio de la Geografía

\begin{tabular}{|l|c|c|c|c|c|c|c|}
\hline & \multicolumn{7}{|c|}{ APARTADO DE LA ENCUESTA EN \% (1) } \\
\cline { 2 - 8 } & $\mathbf{3 . 1}$ & $\mathbf{3 . 2}$ & $\mathbf{3 . 4}$ & $\mathbf{3 . 6}$ & $\mathbf{3 . 7}$ & $\mathbf{3 . 8}$ & $\mathbf{3 . 1 0}$ \\
\hline $\begin{array}{l}\text { Siempre } \\
\text { Mucho }\end{array}$ & 26,44 & 27,80 & 42,62 & 22,81 & 47,33 & 7,47 & 7,2 \\
\hline $\begin{array}{l}\text { A menudo } \\
\text { Bastante }\end{array}$ & 40,33 & 53,53 & 33,61 & 24,69 & 25,93 & 23,24 & 23,87 \\
\hline $\begin{array}{l}\text { A veces } \\
\text { Poco }\end{array}$ & 24,14 & 12,86 & 14,75 & 36,21 & 21,4 & 44,40 & 41,56 \\
\hline $\begin{array}{l}\text { Nunca } \\
\text { Nada }\end{array}$ & 9,20 & 5,81 & 9,02 & 17,28 & 5,35 & 24,9 & 26,75 \\
\hline
\end{tabular}

(1) Apartado 3.1. ¿Te gusta estudiar Geografía?; 3.2. Es necesaria para tu formación; 3.4. El profesorado hace más atractiva la materia; 3.6. Se debería explicar menos y trabajar más en grupos; 3.7. Uso de las TIC; 3.8. Empleo de otras fuentes documentales; 3.10. Sueles hablar de la materia.

A pesar de la ponderación alcanzada en la cuestión anterior, cuando demandamos al alumnado sobre la relevancia de la Geografía para su formación advertimos una tendencia muy positiva en tanto que un 81 ' $33 \%$ de los encuestados responden que es "Muy necesaria" y "Bastante", mientras que lo niegan de forma absoluta (ítem "Nada") únicamente un $5^{\prime} 81 \%$, por debajo del rechazo mostrado en la cuestión precedente. Esta valoración es más significativa entre el alumnado de la ESO que en $2^{\circ}$ de Bachillerato, en donde sólo la juzgan relevante el $43{ }^{\prime} 75 \%$ de los alumnos.

Una cuestión que consideramos esencial es discernir qué contenidos son tratados por la Geografía según los discentes. Para ello se les proponía diversas posibilidades y los resultados han sido muy diversos. Predomina la respuesta "Hechos humanos que tienen una relación con el territorio en el que ocurren" (35'15\% de los encuestados), seguida de la respuesta "Ríos, montañas, capitales, países,..." (18'83\%), "Aspectos económicos, sociales, demográficos de un país" (13'81\%), "Mapas, planos y atlas" (13'39\%) y "El impacto del hombre sobre la naturaleza" (12'13\%). Esto plantea una doble posibilidad: 0 bien no lo tienen muy claro o bien la respuesta depende del nivel en el que se encuentran. Efectivamente, mientras que el ítem de "Hechos humanos..." es mayoritario en $1^{\circ}$ y $2^{\circ}$ de ESO, en $3^{\circ}$ de ESO comienza a tener mayor valoración los ítems "Ríos, montañas,..." 
y “Mapas, planos,...". En $2^{\circ}$ de Bachillerato, aventaja abrumadoramente la de "Ríos, montañas,..." (62'50\%). Creemos que se ha producido un deslizamiento de opiniones en las que la influencia humana era interpretada como más relevante, hacia una concepción de la Geografía como mera relación de aspectos físicos o descriptivos. Nos resulta relevante recalcar este aspecto, puesto que se produce en los niveles educativos donde el currículo es más diversificado y donde se imparte aspectos diversos sobre la Geografía general en $3^{\circ}$ de ESO o la Geografía de España en $2^{\circ}$ de Bachillerato.

Del mismo modo, hemos obtenido resultados muy esclarecedores con la pregunta en la que se cuestiona la influencia del profesorado a la hora de hacer más o menos atrayente la materia, puesto que el 76'23\% de los encuestados (agrupando los ítems "Mucho" y "Bastante") lo creen imprescindible. Indudablemente, la impronta del profesor/a es primordial para atraer a su alumnado a su materia, tanto en Geografía como en otras disciplinas. $\mathrm{Al}$ respecto, hemos de resaltar un aspecto determinante en nuestra disciplina como es la escasa presencia de especialistas en Geografía en la Enseñanza Secundaria. Esto ha provocado que estos contenidos sean impartidos por no especialistas que, en muchas ocasiones, se ven obligados a asumirla sin el máximo entusiasmo posible. Este hecho, tal y como hemos comprobado personalmente en numerosas ocasiones, repercute en la valoración que el alumnado realiza de esta materia.

En cuanto a la opinión que tienen los alumnos/as sobre la cantidad de horas que se le dedica a la Geografía, el 71'72\% del alumnado considera innecesario un incremento o descenso del número de horas (tres en Secundaria o cuatro en Bachillerato).

Sobre la forma de impartir la asignatura, hemos sondeado la opinión del alumnado sobre la siguiente frase "En clase, el profesor/a debería explicar menos y los alumnos/ as trabajar más en grupos". El 53’49\% de los encuestados rechazó esta afirmación. Los resultados varían desde posición totalmente en desacuerdo en $1^{\circ}$ de ESO hasta una apreciación más positiva con esta afirmación en $2^{\circ}$ de Bachillerato. Esto indica que, según avanzamos en niveles educativos, el alumnado se decanta por un aprendizaje menos dirigido y más autónomo.

Uno de los aspectos que están cambiando la forma de impartir las clases, sea cual sea la asignatura de la que se trate, es la introducción de las nuevas tecnologías (ordenadores, móviles, tabletas). Es, por ello, que hemos establecido una cuestión en el cuestionario para averiguar la actitud del alumnado frente al uso de las TIC en nuestra materia. El resultado es que un 73'26\% de los encuestados (agrupando los ítems “Siempre” y “A menudo") juzga muy positivamente el empleo de las TIC, opinión corroborada en otras investigaciones sobre este tema (Seva et alii, 2017). Esta respuesta es mayoritaria en los niveles de ESO en tanto que en $2^{\circ}$ de Bachillerato esta apreciación no es tan nítida. No encontramos un fundamento claro a este resultado salvo, tal vez, el carácter teórico predominante de la materia que prepara para las PEBAU que hace menos interesante el uso de las TIC. 
Hemos ido acreditando hasta ahora que el alumnado se decanta generalmente por la modificación de los roles educativos tradicionales, pero también verificamos que esta afirmación puede trastocarse en el momento en que corresponde al propio alumno tomar la iniciativa y la responsabilidad de un aprendizaje autónomo. Así pues, hemos introducido una cuestión sobre la inclusión de contenidos suplementarios a los temas tratados en clase mediante el uso de internet, enciclopedias, etc. Un 69'30 de los encuestados (agrupando los ítems "A veces" y "Nunca") muestra una negativa a esta posibilidad, rechazo que va incrementándose con los sucesivos niveles hasta llegar al máximo en Bachillerato. Esta misma preferencia apreciamos en el apartado general de hábitos de estudio, y en el cuestionario sobre Historia. Los factores que condicionan esta tendencia pueden ser múltiples y ya hemos apuntado varios en estas páginas, pero podríamos insistir en la existencia de carencias por parte del alumnado en los procedimientos de investigación básicos, mayores dificultades en analizar, comprender, relacionar y sintetizar, de ahí que creemos que el alumnado, en general, se siente más cómodo en la tarea meramente memorística, la tradicional, en la que ha sido formado en la mayor parte de su vida académica y, por consiguiente, menos propicio a cambios en su manera de afrontar el estudio de estas (y otras) asignaturas. De nuevo la influencia del profesorado en cambiar está tónica es esencial.

Del mismo modo, hemos planteado qué temas les gustaría tratar en la clase de Geografía, entre algunos preestablecidos. Así, la gran mayoría de los encuestados ha creído conveniente la realización de un mayor número de salidas didácticas (58'65\%), frente a la introducción de los temas relacionados con la realidad inmediata (24’05\%), la elaboración de trabajos de campo (10’55\%) o la localización de accidentes geográficos en atlas $\left(6^{\prime} 75 \%\right)$.

Finalmente, hemos querido conocer si los alumnos hablan de temas de carácter geográfico con sus compañeros fuera del aula. De forma genérica, podemos confirmar una respuesta negativa, ya que el 68'31\% del alumnado (agrupando los ítems “A veces" y "Nunca") no lo hace habitualmente. Esta cifra es inferior en Bachillerato. No resulta de interés para ellos.

\section{CONCLUSIONES}

Para concluir este trabajo, queremos indicar que, según los datos extraídos de los dos cuestionarios analizados, Historia y Geografía son percibidas por el alumnado de forma muy similar. Hemos evidenciado que aún se mantienen actitudes tradicionales tanto en la enseñanza como en el aprendizaje de las Ciencias Sociales, con un predominio claro de la figura del docente. A medida que madura el estudiante se van adquiriendo hábitos y actitudes diferentes, más abiertos a la gestión individual y autónoma del aprendizaje, 
pero muy limitados por el propio contexto educativo. En cuanto a la Geografía, de manera específica, no existe una gran apreciación por su estudio; se sigue considerando una asignatura poco útil a efectos prácticos, pero importante para la formación del alumnado, esto es, una asignatura más, cuya influencia real va a depender del profesorado y de su capacidad de motivar al alumnado. Creemos que debemos mejorar el proceso de enseñanza de esta materia y para ello es preciso una formación adecuada tanto inicial como permanente del profesorado que la imparte, conocer la asignatura pero también ser capaces de transmitir emociones ya que, como los neurocientíficos afirman, solamente se aprende aquello que emociona y la Geografía tiene capacidad para emocionar y motivar al alumnado. En las manos del profesor/a está transmitir esas emociones.

Sin duda, la enseñanza de las Ciencias Sociales y, particularmente, de la Geografía debe proceder a una actualización no sólo de contenidos (García Pérez, 2017) sino de metodologías para poder acercarla a un alumnado versátil que, en estos momentos, no siente especial predilección por esta materia. Igualmente, creemos necesario promover la autonomía y la creatividad entre el alumnado y utilizar las nuevas tecnologías para respaldar los esfuerzos de cambio que tenemos que afrontar.

Queremos subrayar que conocer la opinión de nuestros alumnos/as es un ejercicio que debemos valorar imprescindible para la mejora de nuestra praxis docente y no sólo como un mero trámite.

Finalmente, nos gustaría agregar que nos hallamos ante un reto muy interesante, un largo camino por recorrer que debemos comenzar lo antes posible si queremos que la Geografía ocupe un lugar destacado entre las diversas materiales que componen el currículum oficial. Sus aportaciones al desarrollo de las competencias clave así lo hacen necesario y conveniente.

\section{BIBLIOGRAFÍA}

Agirre Vicuña, J. y otros, 2013 Mejora de la práctica docente. Una experiencia de autoevaluación. Vitoria-Gasteiz, Servicio de Publicaciones del Departamento de Educación del Gobierno Vasco. [En línea] Disponible en: https://goo.gl/VfiiRv. (Último acceso 28/02/2018).

Alanís Falantes, L. et al. 2016. Nativos digitales y geografía en el siglo XXI: Educación geográfica y sistemas de aprendizaje.A.G.E.: Grupo de Didáctica de la Geografía, Universidad Pablo de Olavide y Universidad de Alicante.

Arroyo Illera, F. 1995. "Una cultura geográfica para todos: El papel de la Geografía en la educación Primaria y Secundaria”, en Moreno Jiménez, A. y Marrón Gaite, Ma J., 1995. 
Benejam, P. y Pagès, J. (1997): Enseñar y aprender Ciencias Sociales, Geografía e Historia en la Educación Secundaria. Barcelona: ICE/Horsori.

Buzo Sánchez, I. - Ibarra Benlloch, P., 2013. Informe "La posición de la Geografía en la Educación Secundaria y Bachillerato”. Edita AGE. [En línea] Disponible en: http://ciug.gal/PDF/informeage13.pdf. (Último acceso 28/02/2018)

Capdevila Gómez, A., Lara Fuillerat, J.M. y Moraga Campos, J. (2016). “Percepción del alumnado sobre la enseñanza y el aprendizaje de la historia”. eCO. Revista Digital de Educación y Formación del profesorado. CEP de Córdoba. [En línea] Disponible en https://wp.me/p8j08t-cG (Último acceso 05/06/2018)

Carretero, M. et al. (comps.), 1997. La enseñanza de las Ciencias Sociales. Madrid: Visor.

Comisión De Educación Geográfica de la UGI, 2016. Declaración Internacional sobre Educación Geográfica. [En línea] Disponible en: http://www.igu-cge.org/charters/ (Último acceso 28/02/018)

De Miguel González, R., 2016. "Pensamiento espacial y conocimiento geográfico en los nuevos estilos de aprendizaje” en Alanís Falantes, L. et al. 2016. Nativos digitales y geografía en el siglo XXI: Educación geográfica y sistemas de aprendizaje. Asociación de Geógrafos Españoles, Grupo de Didáctica de la Geografía, Universidad Pablo de Olavide y Universidad de Alicante, páginas 11-39.

De Miguel, R., De Lázaro, M. L. y Marrón, M. J., eds., 2012. La educación geográfica digital. Zaragoza: Grupo de Didáctica de la Geografía (A.G.E.) y Universidad de Zaragoza.

García Pérez, F. F. 2017. “Algunas reflexiones sobre la trayectoria de la enseñanza de las Ciencias Sociales en las últimas décadas”. eCO. Revista Digital de Educación y Formación del Profesorado. $\mathrm{N}^{\mathrm{o}} 14$. [En línea] Disponible en https://wp.me/p8j08tkB (Último acceso 6/06/2018)

García Ruiz, A. L. y Jiménez López, J.A., 2007. La implementación de los Principios Científicos-Didácticos (P.C.D.) en el aprendizaje de la Geografía e Historia. Granada: EUG.

Graves, N. J., 1989. Nuevo método para la enseñanza de la Geografía. Barcelona: Teide.

Liceras, A. 1997. Las dificultades en el aprendizaje de las ciencias sociales: una perspectiva psicodidáctica. Granada: EUG.

Liceras, A., 2000. Tratamiento de las dificultades de aprendizaje en Ciencias Sociales, Granada: EUG.

Liceras Ruiz, A. y Romero Sánchez, G., Dir., 2016. Didáctica de las Ciencias Sociales. Fundamentos, contextos y propuestas. Madrid: Pirámide. 
López Facal, R. 1992. "Mapas conceptuales y enseñanza de las Ciencias Sociales". Revista Aula de innovación educativa, $n^{\circ}$ 8, noviembre de 1992, pp. 31-35

Moreno Jiménez, A. y Marrón Gaite, Mª J., 1995. Enseñar Geografía. De la teoría a la práctica. Madrid: Síntesis.

Moreno Jiménez, A. 1998. El papel educativo de la Geografía: reflexiones sobre los fines y desafios actuales, Revista da Facultade de Letras-Geografía I serie, Vol. XIV, Porto, págs. 11-37. [En línea] Disponible en: http://ler.letras.up.pt/uploads/ ficheiros/1606.pdf (Último acceso 12/06/2018))

Nin, M. C., y Leduc, S. M. 2013. Leer y comprender en las clases de Geografía. Revista Geograficando, 9 (9). [En línea] Disponible en: https://goo.gl/sS6knC, (Último acceso 6/3/2018)

Prats, J., coord., 2011. Didáctica de la Geografía y la Historia. Barcelona: Graó.

Sebastiá, R. y Tonda, E., eds., 2016: Investigar para innovar en la enseñanza de la Geografía. Alicante: Grupo de Didáctica de la Geografía de la Asociación de Geógrafos Españoles, Universidad de Alicante.

Tourón, J. 2017: “¿Objetivos, estándares, competencias? ¡Aprendizaje para el dominio!”, en blog "Javier Tourón", Enlace: https://goo.gl/U6NdAA. (Último acceso 05/06/2018).

Santiago Rivera, J.A. (2004): "La enseñanza de la Geografía en el mundo global”, Revista Entretemas, No 1, 2004, pp. 101-11. [En línea] Disponible en: https://goo. gl/DM8hzg (Último acceso 11/3/2018)

Seva Cañizares, F., Vera Muñoz, M. I. y Soriano López, M.C., 2017. "La percepción del alumnado de ciencias sociales ante la utilización de las tecnologías de la información y la comunicación en clase. Las TIC favorecen el aprendizaje de las ciencias sociales, según el alumnado” en Martínez Medina, R., García-Morís, R. y García Ruiz, C.R., 2017. Investigación en didáctica de las Ciencias Sociales. Retos, preguntas y líneas de investigación. Córdoba: Universidad de Córdoba y AUPDCS, p. 782-789.

Souto González, J. M., 1998. Didáctica de la Geografía. Barcelona: Ediciones del Serbal.

Vera Muñoz, M.I.y Cubillos Alfaro,F., 2010. "Concepciones del alumnado de secundaria sobre la comprensión y el aprendizaje conceptos de geografía”. Revista Enseñanza de las Ciencia Sociales. Revista de Investigación, $\mathrm{n}^{\circ}$ 9, 3-16. UAB 
